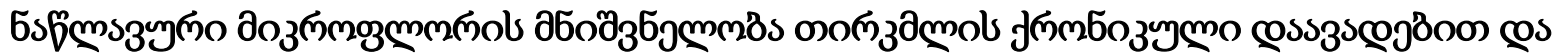

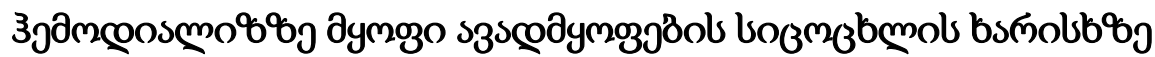

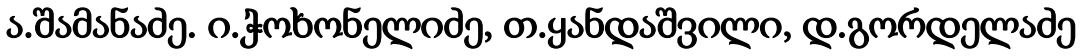

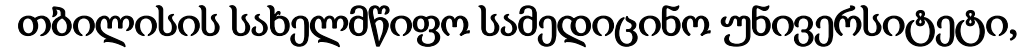

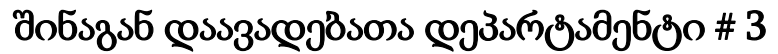

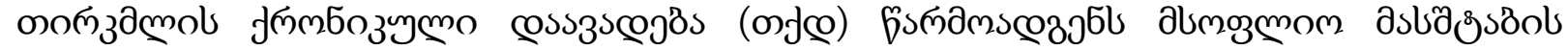

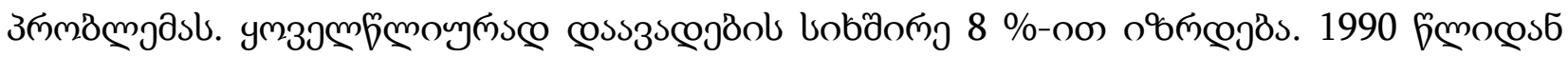

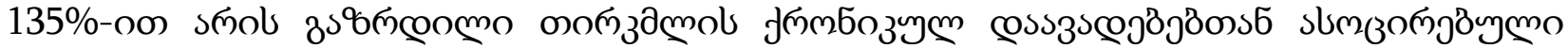

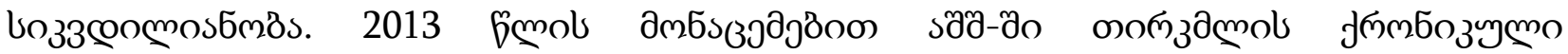

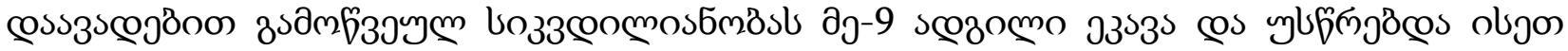

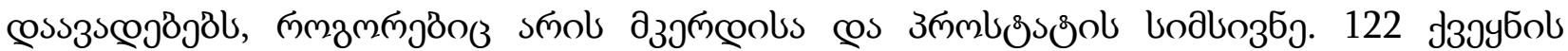

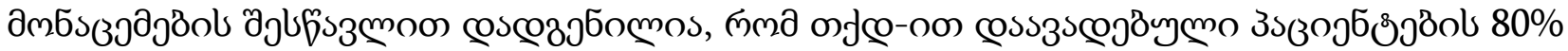

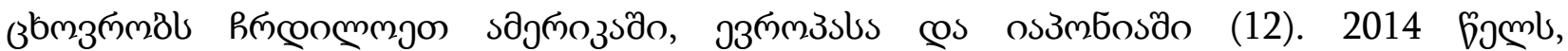

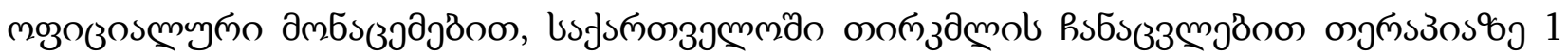

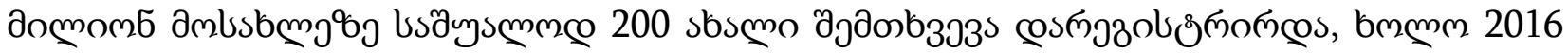

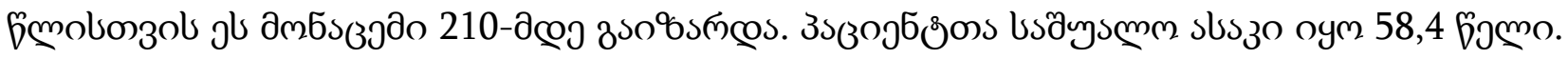

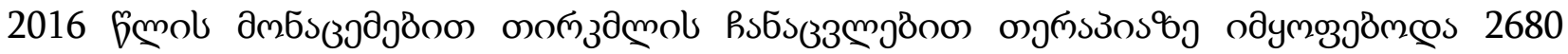

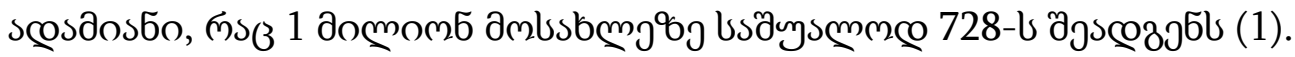

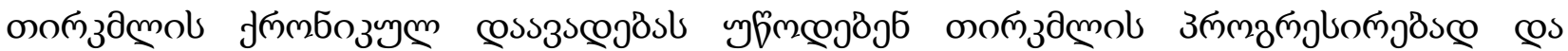

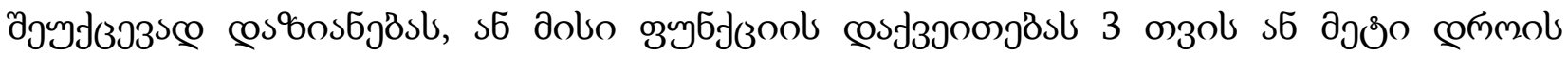

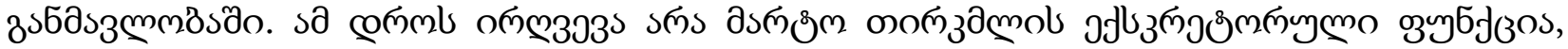

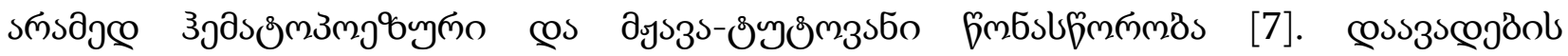

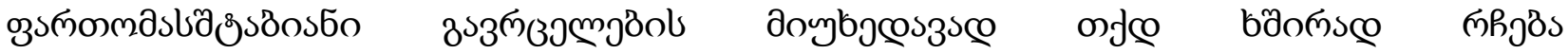

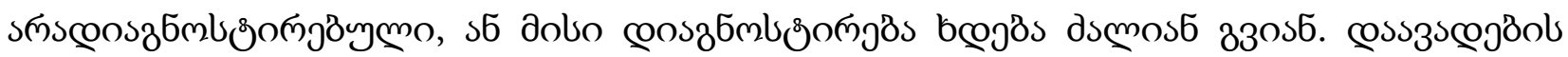

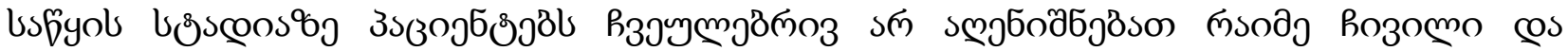

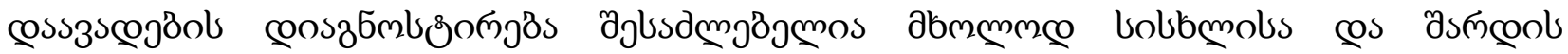

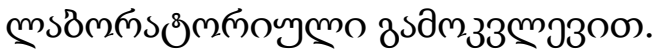

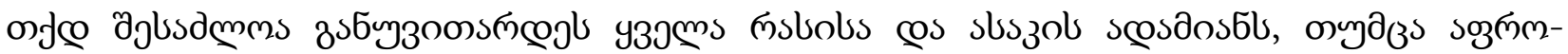

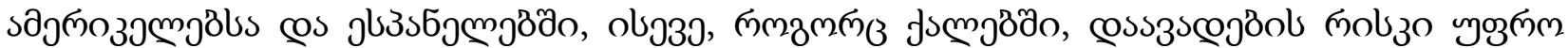

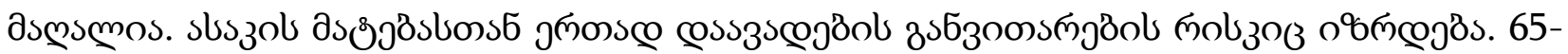

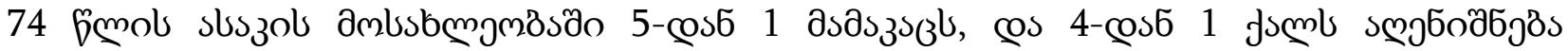

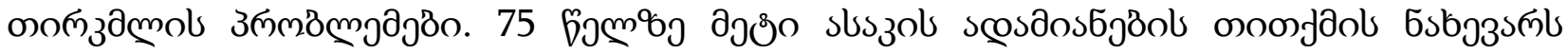

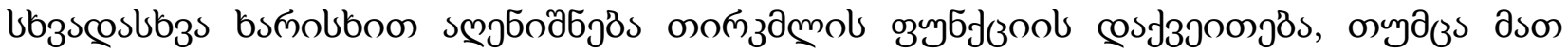

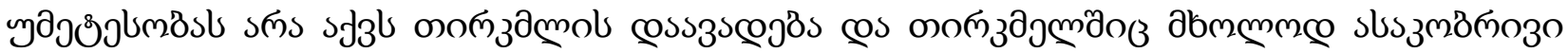

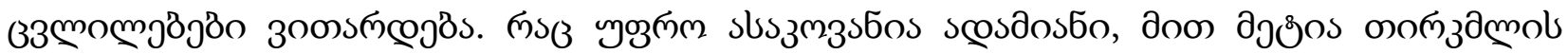

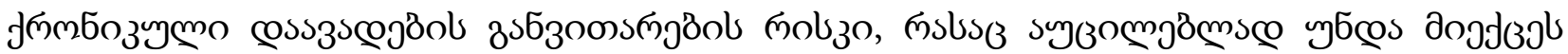

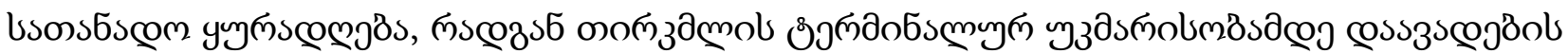

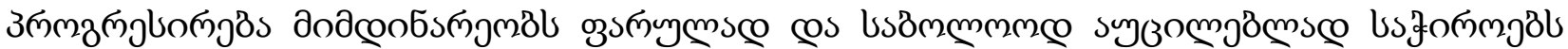

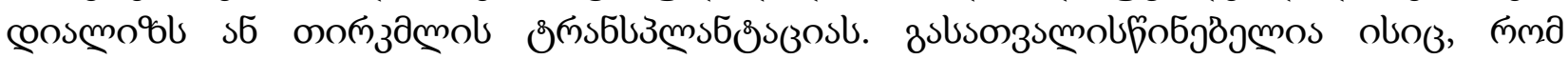




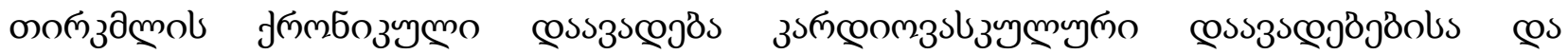

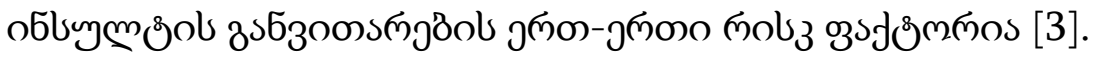

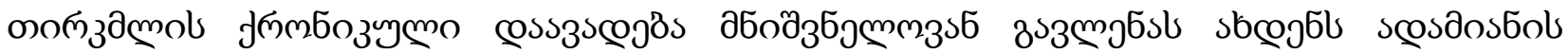

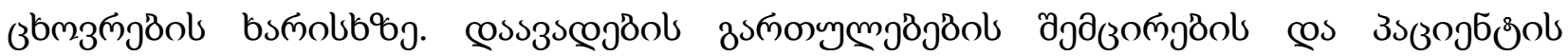

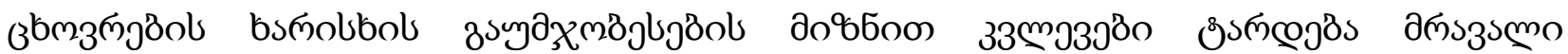

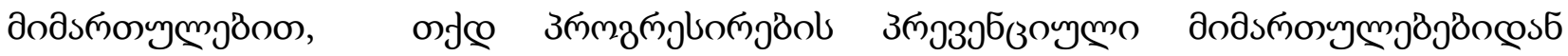

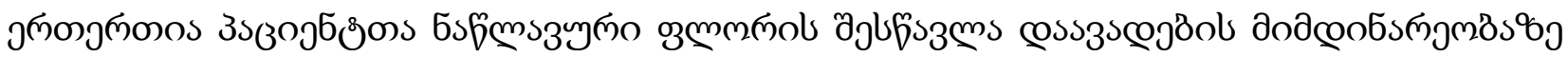

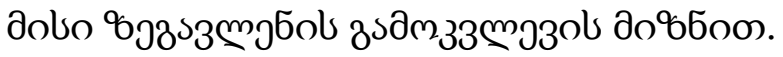

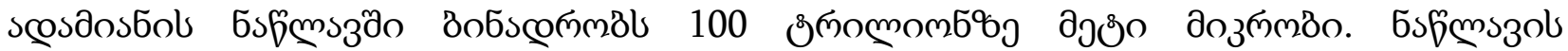

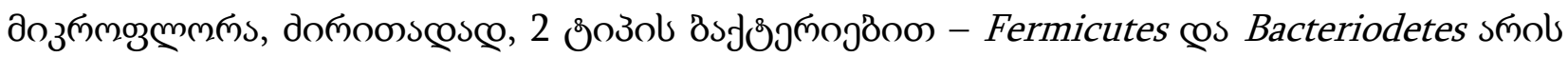

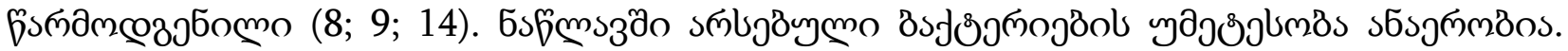

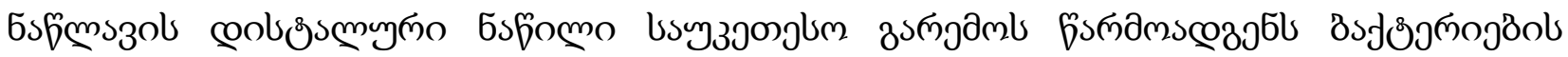

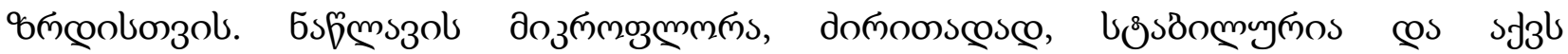

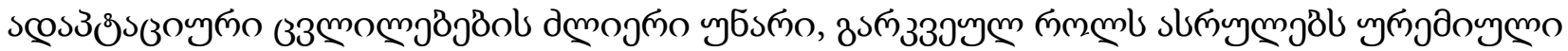

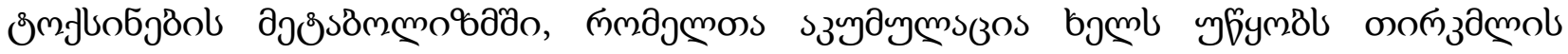

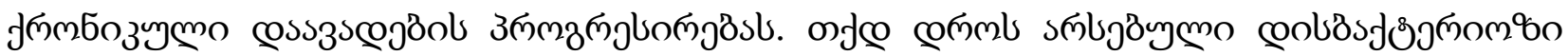

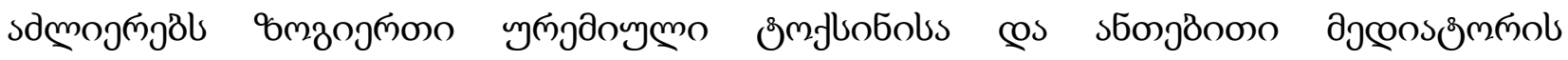

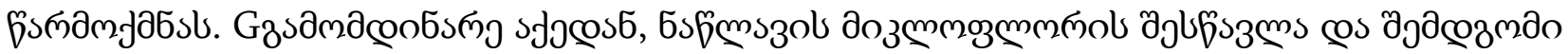

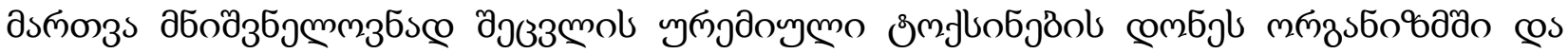

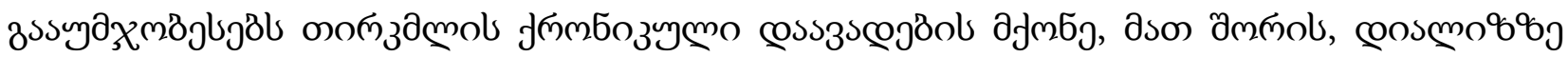

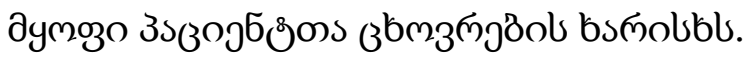

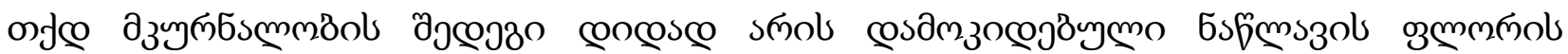

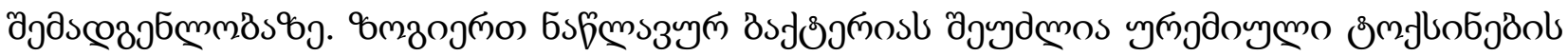

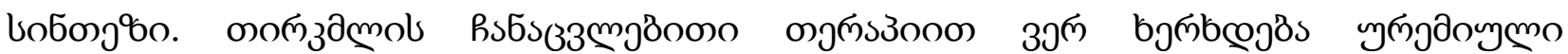

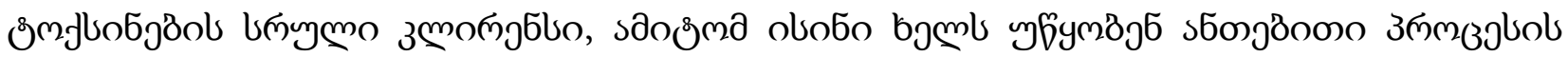

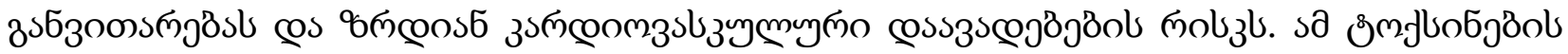

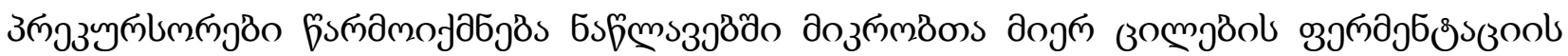

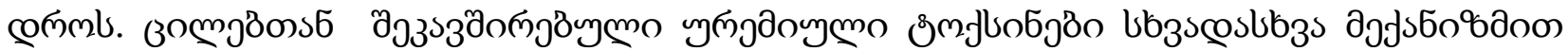

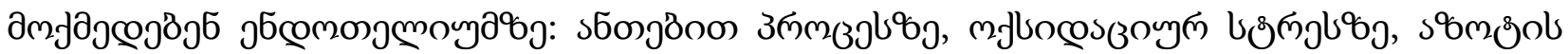

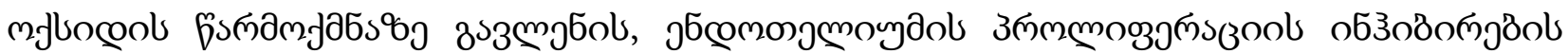

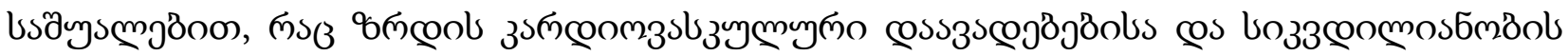

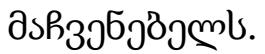

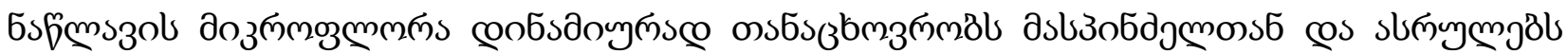

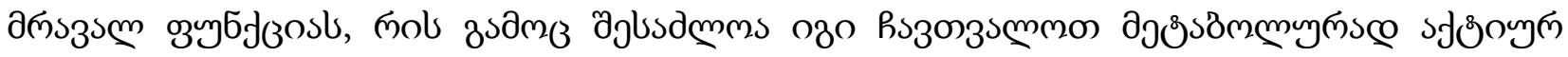

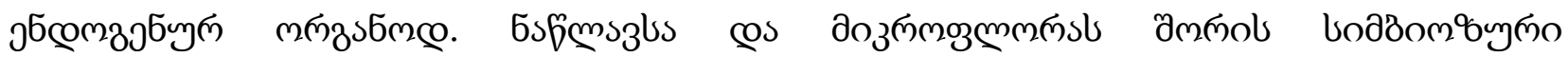

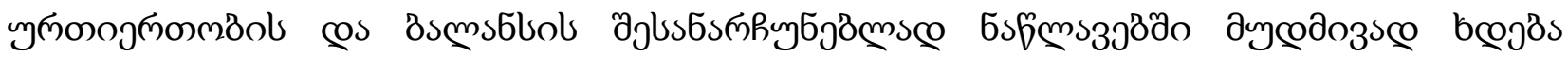

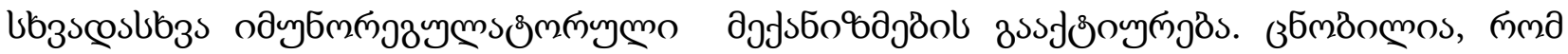

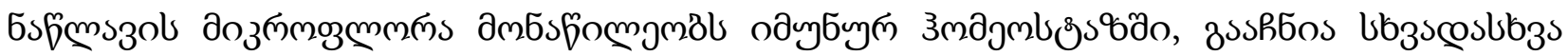

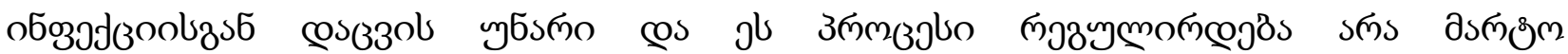

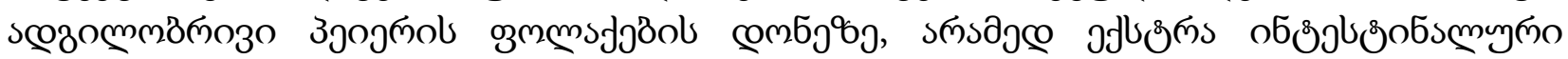

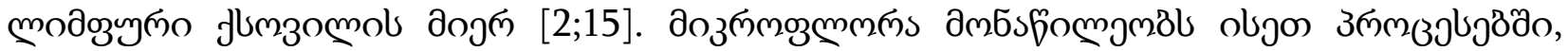

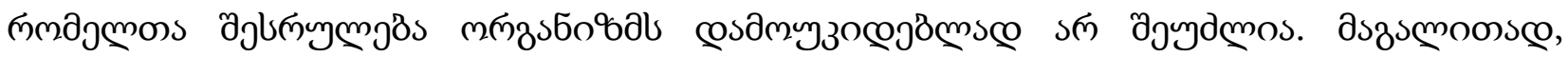

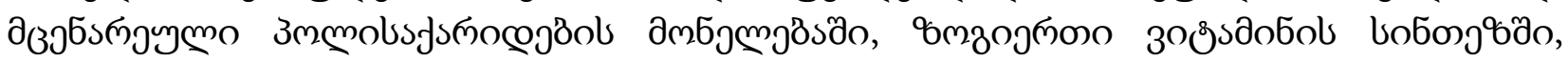




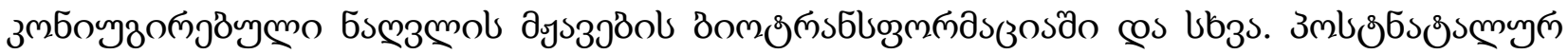

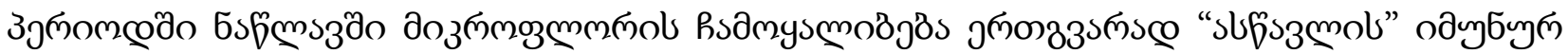

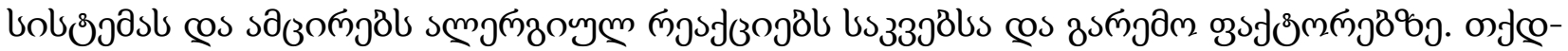

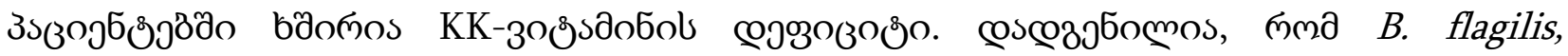
Bifidobacteria, Clostridium @os Streptococcus faecalis dm6sfonmgm

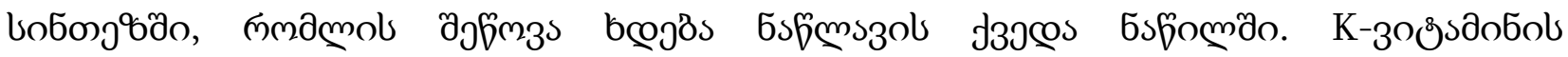

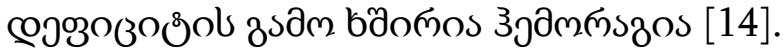

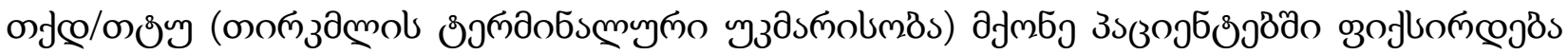

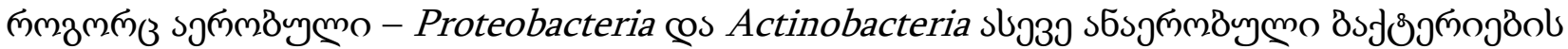

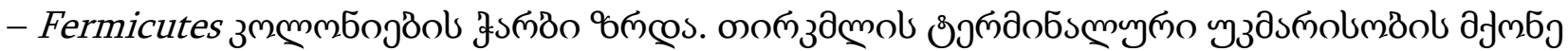

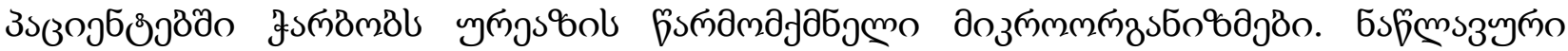

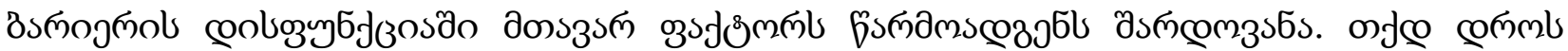

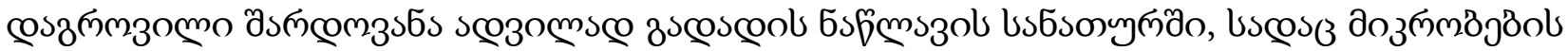

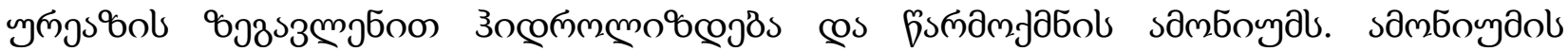

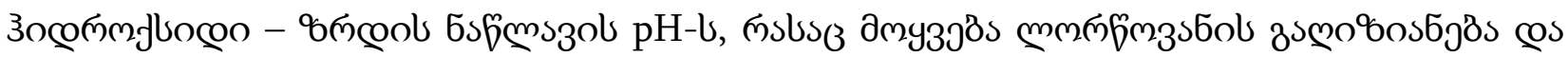

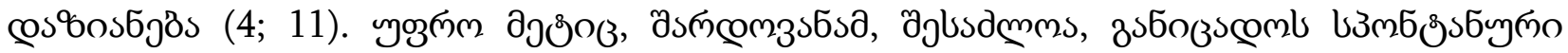

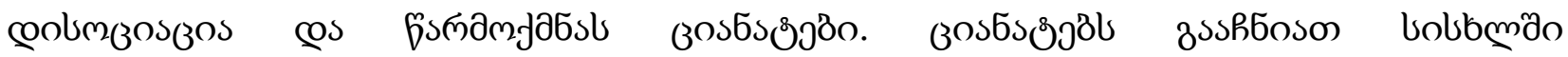

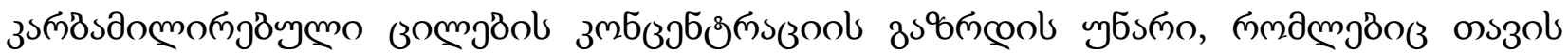

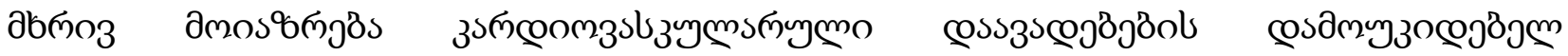

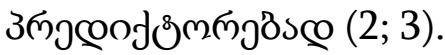

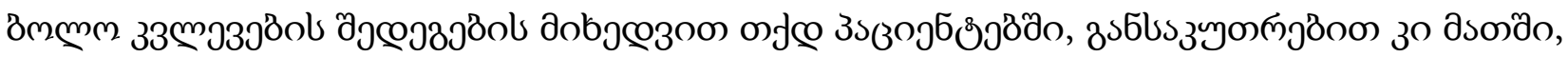

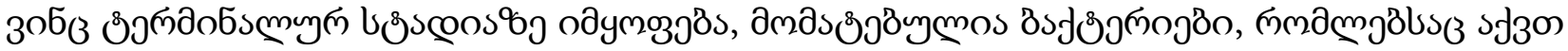

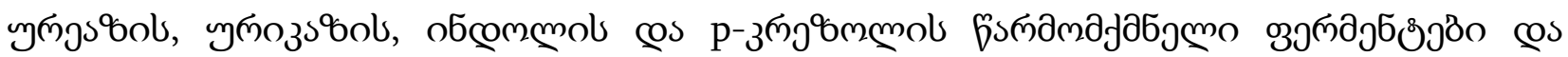

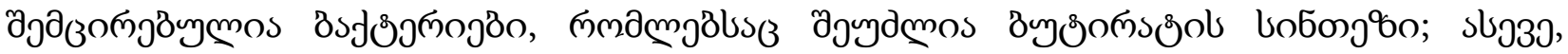

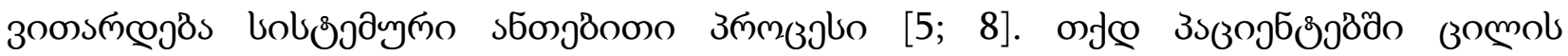

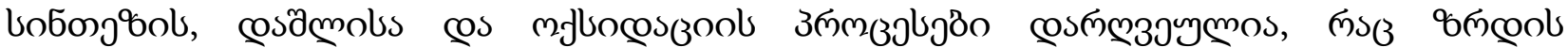

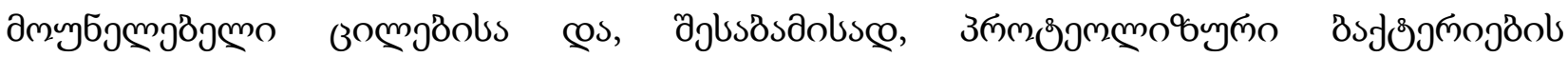

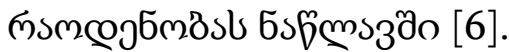

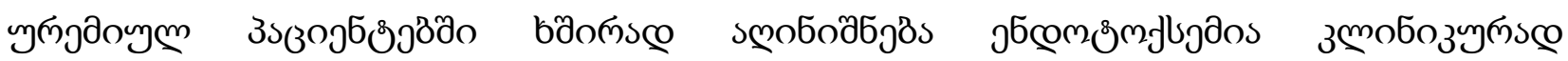

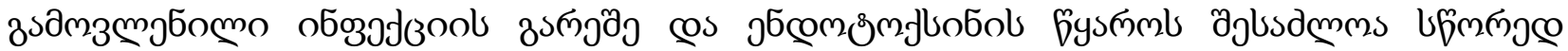

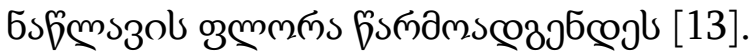

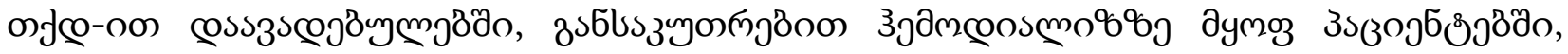

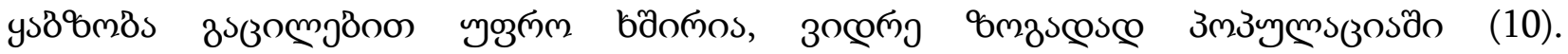

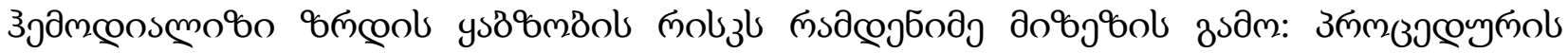

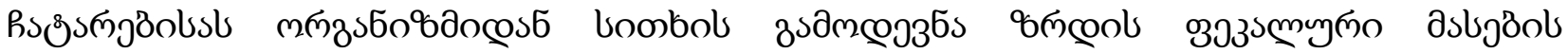

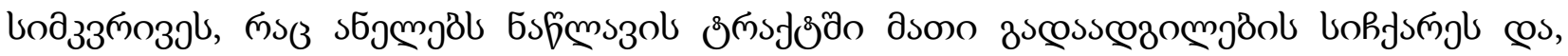

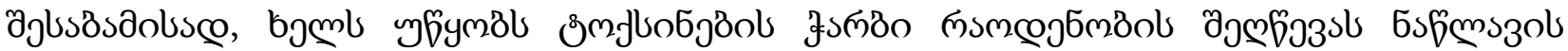

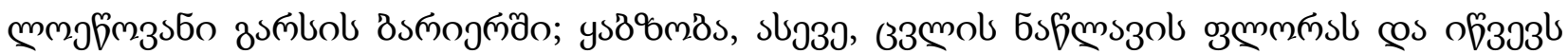

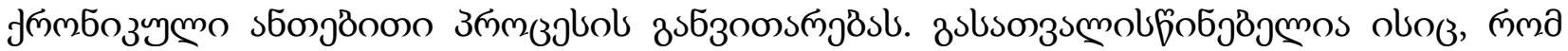

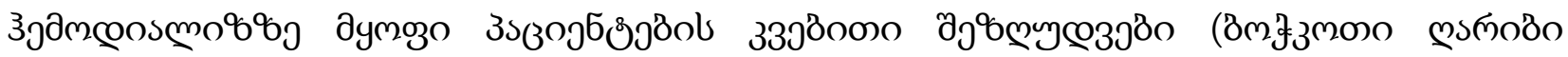

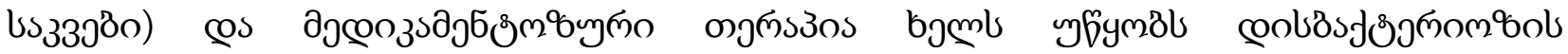

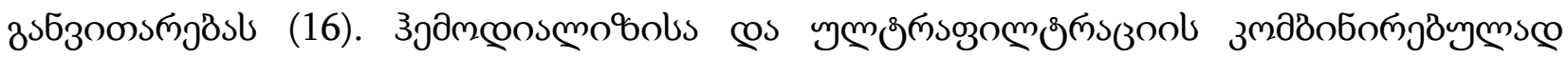

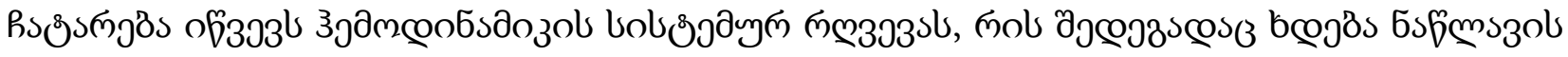

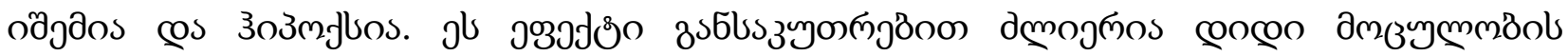




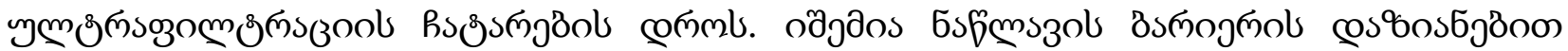

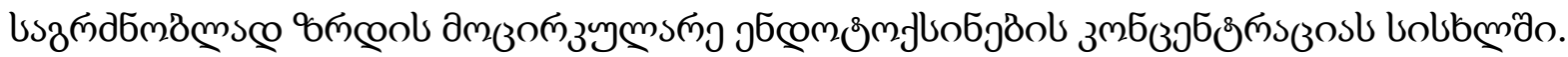

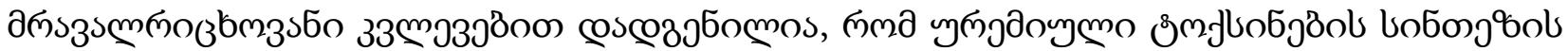

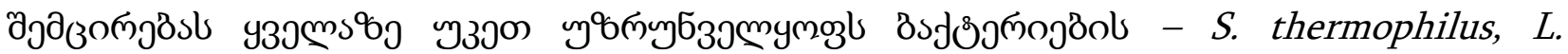
acidophilus, B. longum Әुว

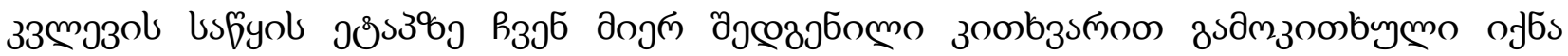

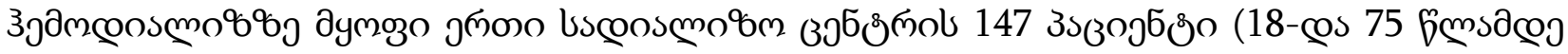

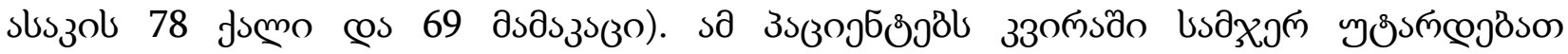

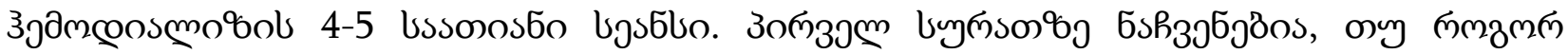

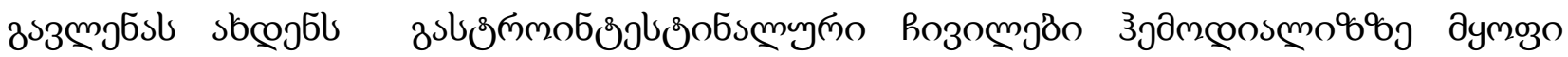

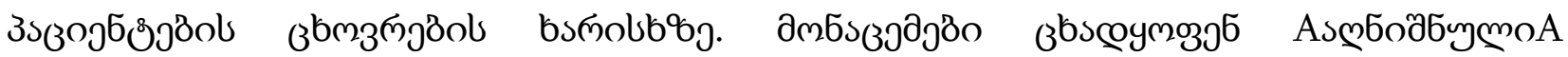

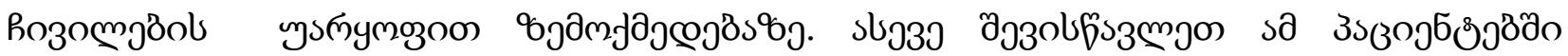

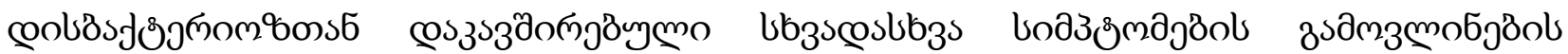

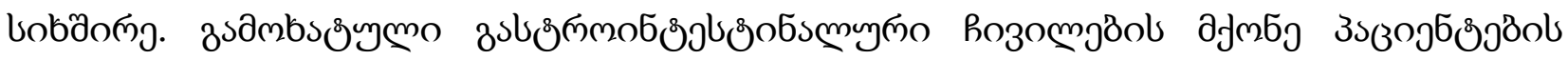

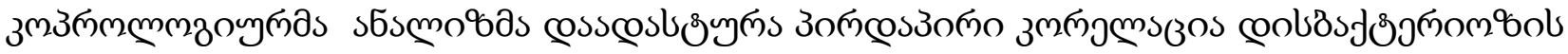

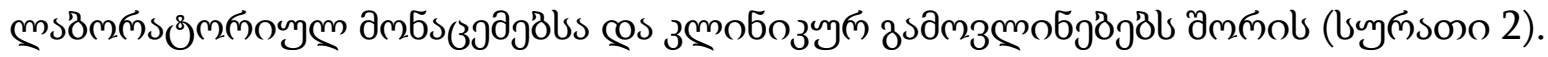

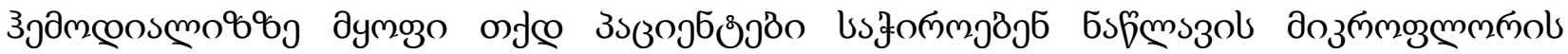

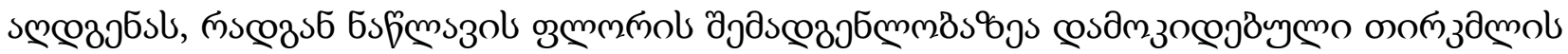

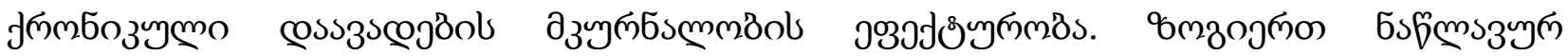

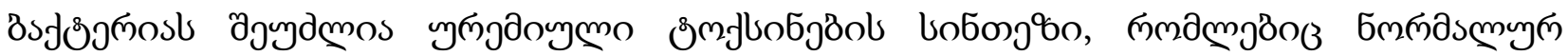

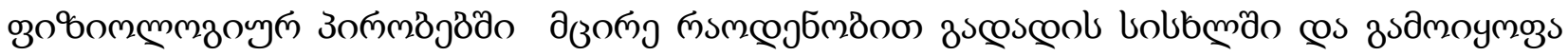

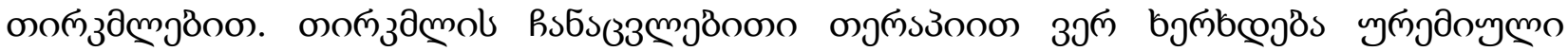

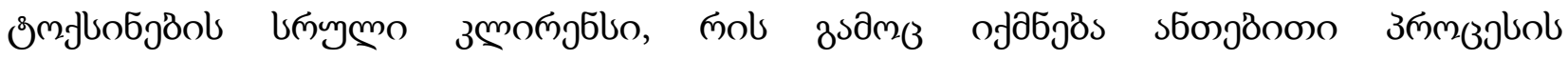

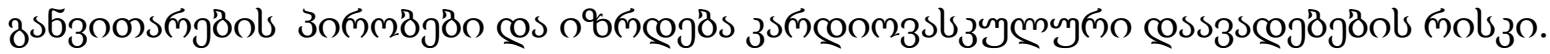

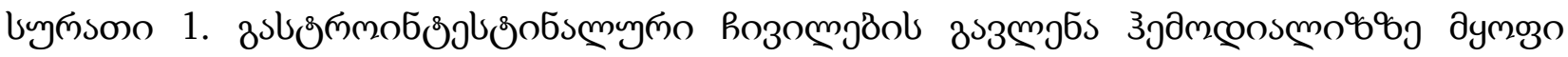

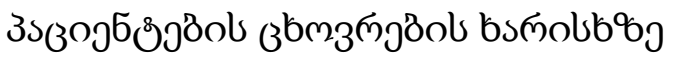

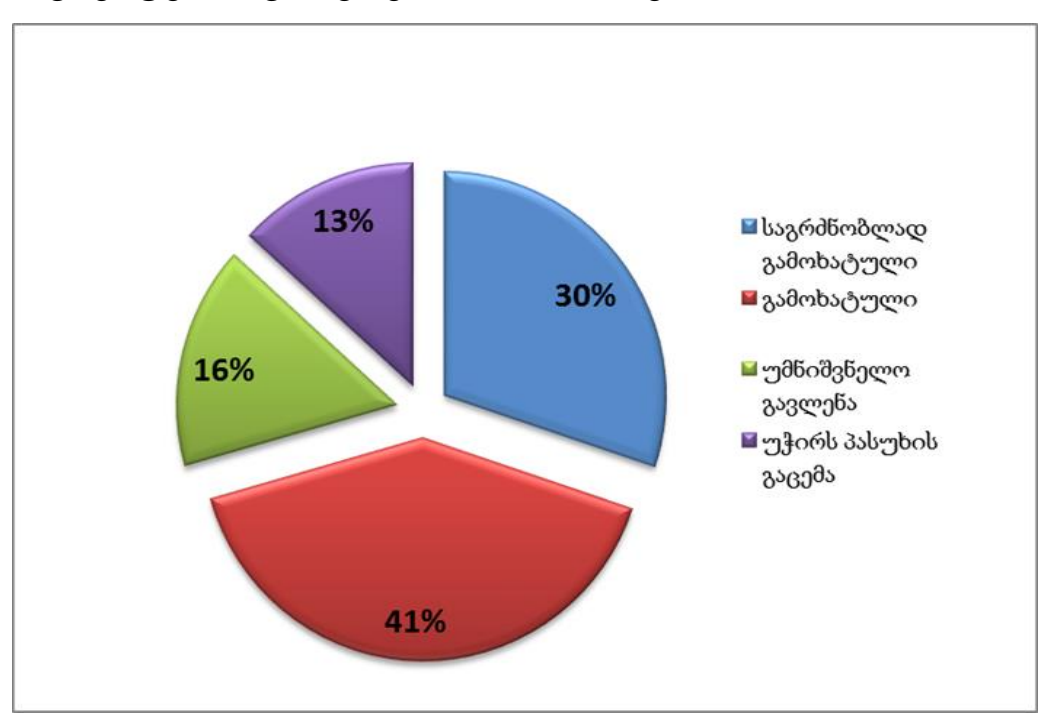




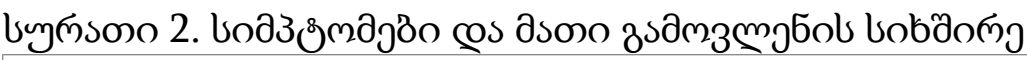

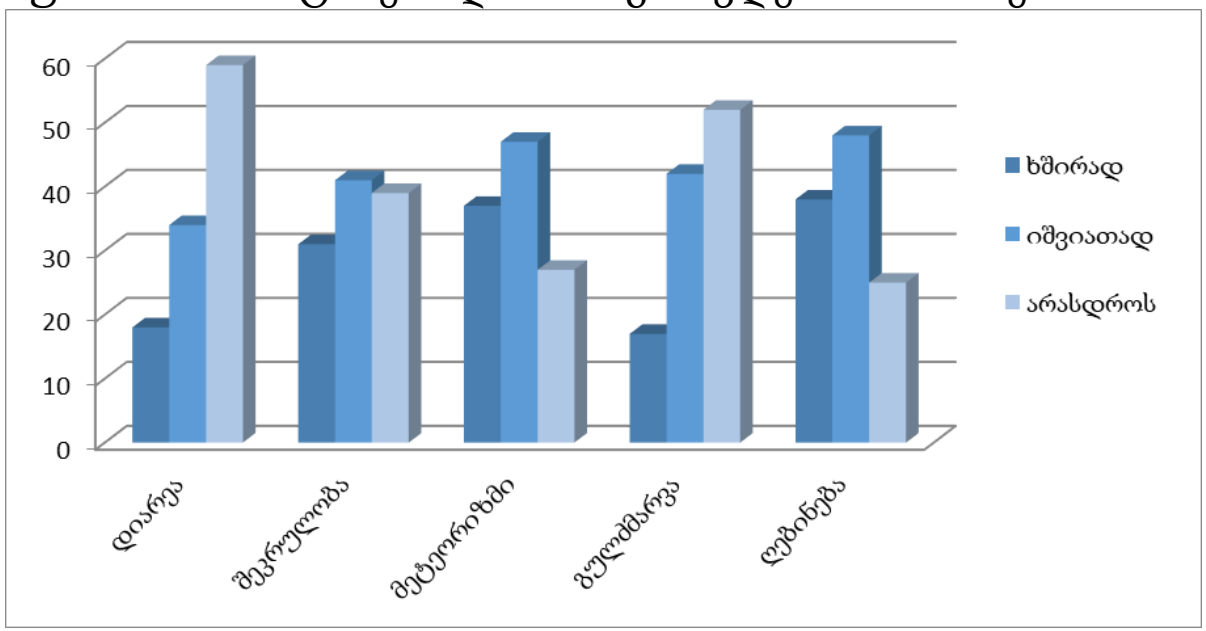

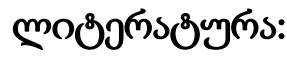

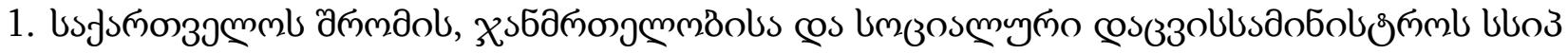

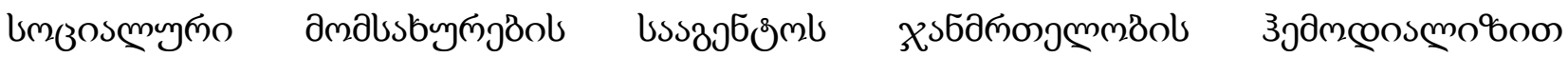

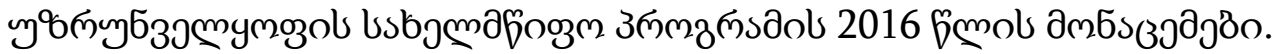

2. Alterations of intestinal barrier and microbiota in chronic kidney disease. Alice Sabatino, Giuseppe golisti, Irene Brusasco, Aderville Cabassi,Santo Morabito and Enrico Fiaccadori.

3. Chronic kidney disease and cardiovascular risk: epidemiology, mechanisms, and prevention Ron T Gansevoort, Ricardo Correa-Rotter, Brenda R Hemmelgarn, Tazeen H Jafar, Hiddo J Lambers Heerspink, Johannes F Mann, Kunihiro Matsushita, Chi Pang Wen. THE Lancet p339-352 July 2013

4. Chronic kidney disease alters the composition of intestinal microbial flora. Kidney Int 2013; 83: 308-315

5. Ckd impairs barrier function and alters microbial flora of the intestine: a major link to inflammation and uremic toxicity, Nosratola D. Vaziri. NIH Public Access 2013 aug

6. Disintegration of colonic epithelial tight junction in uremia: a likely cause of CKD-associated inflammation

7. Essentials of clinical nephrology, 2015.

8. Gut microbiota and inflammation in chronic kidney disease patients. // Denise Mafra and Denis Fouque. Clinical Kidney Journal 2015 may

9. Functional interactions between the gut microbiota and host metabolism. Nature 489: 242249, 2012

10. Intestinal Barrier Disturbances in Haemodialysis Patients: Mechanisms, Consequences, and Therapeutic Options. BioMed Research International Volume 2017 (2016), Article ID 5765417 ,

11. Intestinal-renal syndrome: mirage or reality? Ritz E. 13th International Conference on Dialysis: Advances in Chronic Kidney Disease 2011

12. The contribution of chronic kidney disease to the global burden of major noncommunicable diseases. Couser WG, Remuzzi G, Mendis S, Tonelli .

13. The gut as a source of inflammation in chronic kidney disease. Wei Ling Lau, Kamyar Kalantar-Zadeh, Nosratola D. Vaziri. Nephron Clinical practice NCBI, 2015 
14. The Gut Microbiome, Kidney Disease, and Targeted Interventions. Ali Ramezani, Dominic S. Raj. Journal of the American Society of Nephtology 2014

15. The intestinal microbiota, a leaky gut, and abnormal immunity in kidney disease. Anders HJ, Andersen K, Stecher B. Journal of Nephrology and Therapeutics june 2013,

16. The Kidney-Gut Axis: Implications for Nutrition Care. Journal of Renal Nutrition 2015 sept

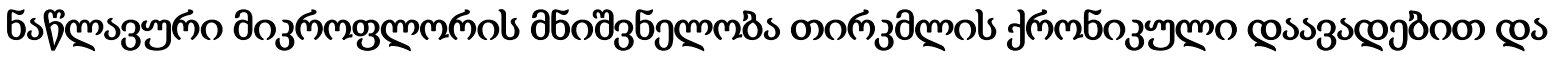

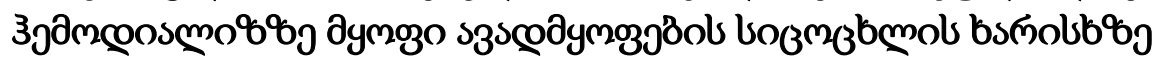

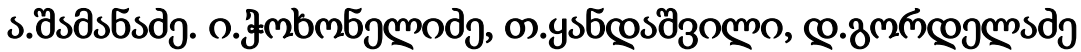

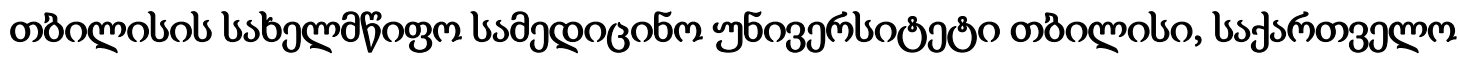

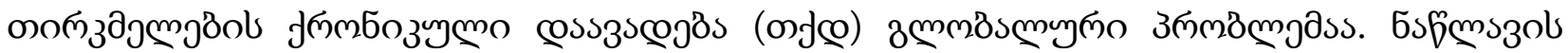

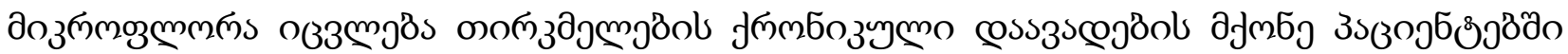

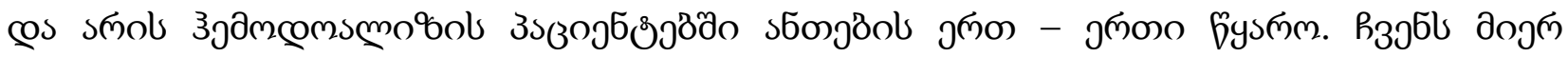

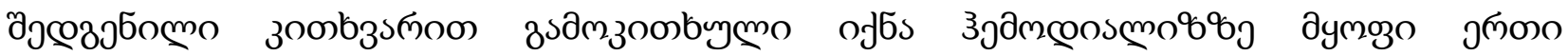

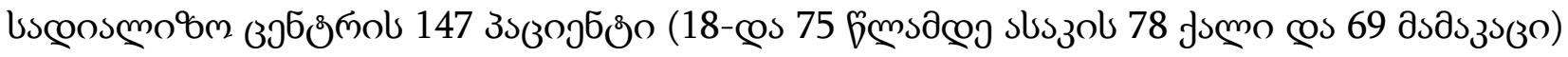

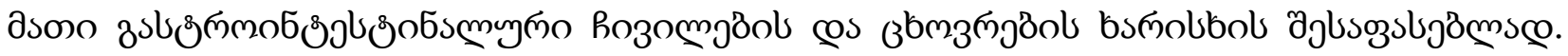

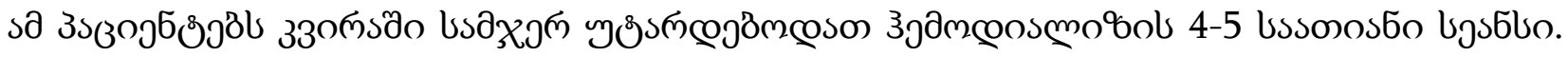

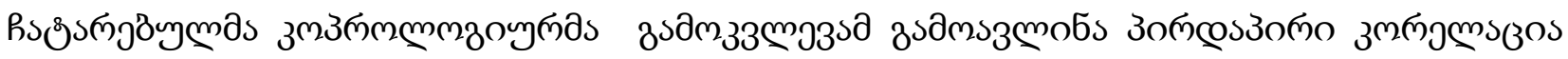

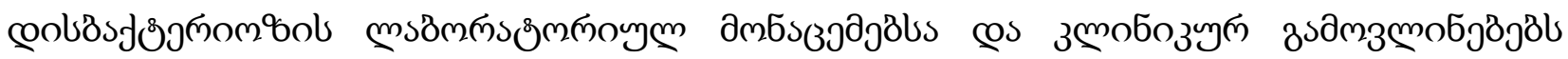

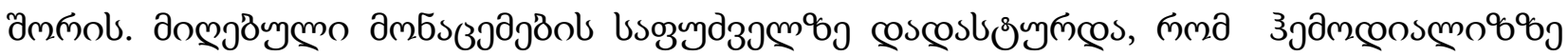

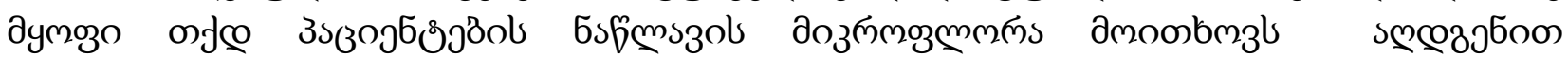

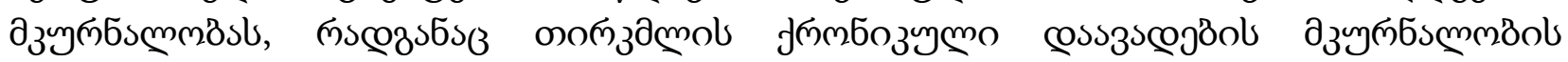

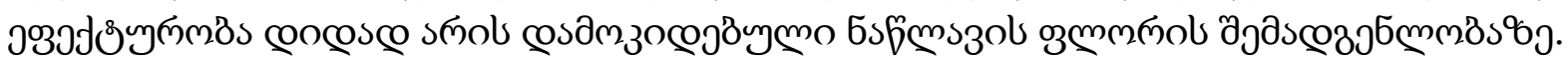




\section{Impact of Intestinal Microbiota on Quality of Life (QoL) of Hemodialysis Patients}

\section{A. Shamanadze; T. Kandashvili; I. Tchokhonelidze; D. Gordeladze}

\section{Tbilisi State Medical University Tbilisi, Georgia.}

Chronic kidney disease is a global problem. Increasing evidence suggests that interstinal microflore is altered in patients with Chronic Kidney Disease (CKD).The gut microbiome is a key driver of chronic inflammation. Many of uremic retention solutes are produced by dysbiotic intestinal microflore that may have a great impact on survival of the CKD patients.

Our sample study consisted of 147 patients (78 female, 69 male age 18-75) undergoing hemodialysis in one dialysis center. Data were collected by the completion of a scpecially designed questionnaire. Patients received hemodialysis therapy for 4-5 hours sessions, three times weekly using polysulfone dialyzers. It was revealed significant alteration of the colonic bacterial flora in hemodialysis patients, with the excess of the uremic toxin producing bacteria. Also, there was strict correlation between gut dysbiosis and HD patent's QoL. 